\title{
Coating of Gibbsite Platelets with Silica
}

\author{
J udith E. G. J . Wijnhoven ${ }^{\dagger}$ \\ Van 't H off Laboratory for Physical and Colloid Chemistry, Debye Institute, Utrecht \\ University, Padualaan 8, $3584 \mathrm{CH}$ Utrecht, The Netherlands
}

Received April 2, 2004. Revised Manuscript Received J uly 8, 2004

\begin{abstract}
In this work, gibbsite particles are synthesized from aluminum alkoxides in an acidic environment at $85^{\circ} \mathrm{C}$. After centrifugation, coating with silica is investigated following two different methods. Practical aspects and the feasibility to produce significant amounts of material are studied. The first method, analogous to a procedure used for boehmite, is a three-step procedure that starts from the addition of water glass and ends with Stöber growth of silica. In the second method, poly(vinylpyrrolidone) is used to stabilize gibbsite in the Stöber-like mixture for silica growth. Both methods lead to silica-coated particles. The first method is elaborate, but can be used to make very thin $(<4 \mathrm{~nm})$ silica layers. The second method is quick and produces smooth layers. The particles are characterized by scanning and transmission electron microscopy and energy-dispersive X-ray analysis and show a smooth silica layer of 10-25 nm thick. These particles can be used for confocal microscopy and in mixtures with different shaped particles to study phase behavior. By acid leaching the gibbsite can be removed and hollow silica platelets remain.
\end{abstract}

\section{Introduction}

Gibbsite $\left(\gamma-\mathrm{Al}(\mathrm{OH})_{3}\right)$ is the main part of the aluminum ore bauxite. ${ }^{1}$ In that quality its processing (Bayer process) is studied frequently. ${ }^{2-4}$ Its surface behavior is also studied extensively as gibbsite is an important part of soils.5,6 Recently, gibbsite proved to be very suitable as a platelike model colloid; its plates are quite monodisperse and easy to synthesize. The dynamics of suspensions of polyisobutene-coated plates was studied. Information about sedimentation and diffusion behavior and the rheological properties has been obtained as a function of volume fraction. ${ }^{7}$ Liquid crystal phase transitions ${ }^{8}$ and the phase behavior of mixtures of plates and rods have also been investigated. ${ }^{9}$ By adding al umina chlorohydrate $(\mathrm{ACH})$, the problem of gelation, common in charged colloidal systems, can be overcome. Particles covered with $\mathrm{ACH}$ are still charged, and therefore, the phase behavior of particles with longrange interactions can be studied. ${ }^{10}$ To be able to extend

† E-mail: j.e.g.j.wijnhoven@chem.uu.nl.

(1) Hind, A. R.; Bhargava, S. K.; Grocott, S. C. Colloids Surf., A 1999, 146, 359-374.

(2) Sweegers, C.; de Coninck, H. C.; Meekes, H.; van Enckevoort, W. J. P.; Hiralal, I. D. K.; Rijkeboer, A. J . Cryst. Growth 2001, 233, 567-582.

(3) Addai-Mensah, J .; Prestidge, C. A.; Ralston, J . Miner. Eng. 1999, $12,655-669$

(4) Prestidge, C. A.; Ametov, I.; Addai-Mensah, J . Colloids Surf., A 1999, 157, 137-145.

(5) Gimsing, A. L.; Borggaard, O. K.; Sestoft, P. Environ. Sci. Technol. 2004, 38, 1718-1722.

(6) Hiemstra, T.; Yong, H.; van Riemsdijk, W. H. Langmuir 1999, 15, 5942-5955.

(7) van der Kooij, F. M.; Philipse, A. P.; Dhont, J . K. G. Langmuir 2000, 16, 5317-5323.

(8) van der Kooij, F. M.; Kassapidou, K.; Lekkerkerker, H. N. W. Nature 2000, 406, 868-871.

(9) van der Kooij, F. M.; Lekkerkerker, H. N. W. Phys. Rev. Lett. 2000, 84, 781-784.

(10) van der Beek, D.; Lekkerkerker, H. N. W. Europhys. Lett. 2003. the phase behavior studies to mixtures of plates and spheres or rods, which are more comparable to natural systems, it is necessary that all the particles have the same surface properties. Silica is known to have hard interactions, a first step to applications. ${ }^{11}$ Since rods can be coated with silica and silica spheres are also available, silica is a good choice for a new coating for gibbsite.

The aim of the research described in this paper is to obtain platelike gibbsite particles in the colloidal size range coated with silica. The aspect ratio of the particles will be variable, depending on the thickness of the silica layer. These particles are meant to be used in phaseseparation experiments. Several methods to produce colloidal gi bbsite particles exist. ${ }^{12-17}$ We chose a method with a relative low polydispersity. This method was developed by Wierenga et al. ${ }^{17}$ and produces gibbsite platel ets with an average diameter of 150-200 nm. Up to now, it has not been possible to follow the phase separation of the samples in situ. To be able to do so, e.g. by confocal microscopy, dye should be incorporated in the particles. Another advantage of silica coating is now present: dye can be incorporated in silica. ${ }^{18}$

(11) Imhof, A.; Dhont, J . K. G. Phys. Rev. Lett. 1995, 75, 16621665

(12) Gastuche, M. C.; Herbillon, A. Bull. Soc. Chim. 1962, 14041412.

(13) Lee, Y.-P.; Liu, Y.-H.; Yeh, C.-S. Phys. Chem., Chem. Phys. 1999, 1, 4681-4686.

(14) Phambu, N.; Humbert, B.; Burneau, A. Langmuir 2000, 16, 6200-6207.

(15) Rasmussen, D. H.: Brancewicz, C.; Das, B.: Graeffe, M. Rosenholm, J .; Toscano, A. J . Dispersion Sci. Technol. 2001, 22, 491498.

(16) Rosenqvist, J .; Persson, P.; Sjoberg, S. Langmuir 2002, 18, $4598-4604$.

(17) Wierenga, A. M.; Lenstra, T. A.J J; Philipse, A. P. Colloids Surf., A 134, 359-371.

(18) van Blaaderen, A.; Vrij, A. Langmuir 1992, 8, 2921-2931. 
Various studies report on the coating of different kinds of colloids with silica: gold, ${ }^{19}$ silver, ${ }^{20}$ boehmite needles, ${ }^{21,22}$ magnetic particles, ${ }^{23,24}$ and polystyrene. ${ }^{25,26}$ With silica coating one is able to control optical properties, ${ }^{19,20}$ tune the interaction of the cores by varying the silica layer thickness, ${ }^{21,23}$ al ter the stability in different solvents, ${ }^{21,22}$ and produce hollow silica particles after removal of the core. ${ }^{25,26}$ But coating with silica also has other advantages. The coating is widely applicable, which makes it easier to work with particles of different shapes. A lot is known about the chemistry of colloidal silica spheres, knowledge that can be applied to the silica-coated particles as well. ${ }^{21} \mathrm{~F}$ or example, fluorescent dyes might be incorporated into silica layers. ${ }^{18}$ Fluorescent particles can be studied by confocal microscopy. This makes it possible to observe the phase separation behavior in situ. Procedures for silica coating vary widely, depending on the type of particle. The easiest way to grow silica layers is by means of the Stöber method. ${ }^{27,28}$ In the Stöber synthesis, silica is grown in a mixture of ethanol, ammonia, and water, to which tetraethoxysilane (TEOS) is added. This method produces smooth layers because growth takes place on a molecular scale. The thickness of the layers can easily be tuned by adding the proper amount of TEOS. All the known silica chemistry, such as the incorporation of dye ${ }^{18}$ or surface modifications of silica with stearyl al cohol, ${ }^{29}$ polyisobutene, ${ }^{30}$ or TMP ${ }^{31}$ may be used.

In case the particles are not stable in the Stöber mixture, an intermediate step in which the particle surface is modified is used. This modifying step can be a coating with, for example, sodium silicate solution (water glass), ${ }^{22}$ poly(vinylpyrrolidone), ${ }^{32}$ (PVP) or (3aminopropyl)trimethoxysilane (APS). ${ }^{19}$ Gibbsite is not stable in ethanol, so surface modification is required. Here, the modification of gibbsite with either sodium silicate solution or PVP is reported.

\section{Experimental Section}

2.1. Gibbsite Synthesis. Gibbsite was synthesized according to the method described by Wierenga et al. ${ }^{17}$ Typically, in $2 \mathrm{~L}$ of double distilled water acidified by $0.06 \mathrm{~mol} / \mathrm{L}(5.1 \mathrm{~g} / \mathrm{L})$ $\mathrm{HCl}(37 \%$ p.a., Merck), $0.08 \mathrm{~mol} / \mathrm{L}(19.7 \mathrm{~g} / \mathrm{L})$ aluminum secbutoxide (ASB, $\geq 95 \%$, Fluka) and $0.08 \mathrm{~mol} / \mathrm{L}(16.3 \mathrm{~g} / \mathrm{L})$ aluminum isopropoxide (AIP, 98+ \%, Acros) were dissolved

(19) Liz-Marzan, L. M.; Giersig, M.; Maulvaney, P. Langmuir 1996, 12, 4329-4335.

(20) Hardikar, V. V.; Matijevic, E. J . Colloid Interface Sci. 2000, 221, 133-136.

(21) van Bruggen, M. P. B. Langmuir 1998, 14, 2245-2255.

(22) Phillipse, A. P.; Nechifor, A.-M.; Patmamanoharan, C. Langmuir 1994, 10, 4451-4458.

(23) Philipse, A. P.; Bruggen, M. P. B. v.; Pathmamanoharan, C. Langmuir 1994, 10, 92-99.

(24) Ohmori, M.; Matijevic, E. J . Colloid Interface Sci. 1992, 150, 594-598.

(25) Caruso, F.; Caruso, R. A.; Moewald, H. Chem. Mater. 1999, $11,3309-3314$

(26) Caruso, F.; Caruso, R. A.; Moehwald, H. Science 1998, 282, $1111-1114$

(27) Bogush, G. H.; Tracy, M. A.; Zukoski, C. F. J . Non-Cryst. Solids 1988, 104, 95-106. 69

(28) Stoeber, W.; Fink, A. J . Colloid Interface Sci. 1968, 26, 62-

(29) van Helden, A. K.; J ansen, J . W.; Vrij, A. J . Colloid Interface Sci. 1981, 81, 354-368.

(30) Pathmamanoharan, C. Colloids Surf. 1988, 34, 81-88.

(31) Philipse, A. P. J . Colloid Interface Sci. 1987, 128, 121-136.

(32) Graf, C.; Vossen, D.; I mhof, A.; van Blaaderen, A. Langmuir 2003, 19 (17), 6693-6700. by stirring for 7-10 days. The resulting solution, which varied from al most clear to turbid, was heated in a polypropene bottle in a water bath at $85{ }^{\circ} \mathrm{C}$ for $72 \mathrm{~h}$. After cooling to room temperature, the dispersion was dialyzed 1 week against a continuous flow of demineralized water in regenerated cellulose tubes (Visking, MWCO 12 000-14 000). The dispersion was centrifuged to remove very small gibbsite particles and other alumina sols, before characterization and use.

2.2. Silica Coating: Water Glass Method. Gibbsite particles were coated with silica by a three-step method initially developed for boehmite. ${ }^{21}$ In the first step, an $1 \mathrm{~L}$ flask of a $5 \mathrm{~g} / \mathrm{L}$ dispersion of gibbsite particles was placed in a tapwater-cooled ultrasonic bath.

A $3 \%$ solution of water glass, sodium silicate $\left(\mathrm{Na}_{2} \mathrm{Si}_{3} \mathrm{O}_{7}\right.$ pure, Riedel-de Haën), was set at $\mathrm{pH}=11$ by adding a small amount of Dowex ion-exchange resin ( $50 \mathrm{~W} \times 8$, Fluka). B efore use, Dowex was washed several times with $\mathrm{HCl}$, methanol, and water to exchange $\mathrm{Na}^{+}$for $\mathrm{H}^{+}$and to clean the resin. The water glass was added to the dispersion at a rate of $3.0 \mathrm{~mL} / \mathrm{min}$ while the dispersion was stirred to mix the water glass (set at a pH of 11) quickly and thoroughly throughout it. Ultrasonification is needed at the same time to avoid aggregate formation during the change of the $\mathrm{pH}$. After $16 \mathrm{~min}$, the flow rate was diminished to $2.1 \mathrm{~mL} / \mathrm{min}$ for another $16 \mathrm{~min}$.

During the last $8 \mathrm{~min}$ the flow rate was $1.0 \mathrm{~mL} / \mathrm{min}$. During the addition of the water glass the $\mathrm{pH}$ was checked regularly. By adding small amounts of Dowex, the $\mathrm{pH}$ was kept below $10 \mathrm{in}$ order to prevent dissol ution of the silica particles formed from the water glass. After the addition of the water glass, the dispersion was stirred for half an hour without ultrasonification. The resulting white dispersion was filtered to remove the Dowex and dialyzed for 9 days to remove the remaining nonadsorbed water glass. Dialyzing for too long a period will lead to the removal of the silica from the gibbsite particles. By use of TEM, the formation of undesired silica particles was checked. They should be removed by centrifugation before starting the next coating step.

As the water glass only formed one thin layer of silica, a second silica layer was applied by adding small amounts of TEOS (pure, Fluka), to a dispersion with a pH of 8-9. To 1.5 $\mathrm{L}$ of a $5 \mathrm{~g} / \mathrm{L}$ water-glass-coated gibbsite dispersion in water, ammonia (p.a. Merck, 25 w/w \%) of pH 10 was carefully added until the desired $\mathrm{pH}$ was obtained. Every $2 \mathrm{~h}, 0.24 \mathrm{~mL}$ of TEOS was added under the surface of the gibbsite dispersion. Meanwhile, the dispersion was stirred vigorously to emulsify the TEOS quickly. This procedure was repeated eight times. The dispersion was subsequently centrifuged for $60 \mathrm{~h}$ at $330 \mathrm{~g}$ and redispersed carefully in a mixture of water and ethanol (50/50 v/v\%) to a total volume of $1.5 \mathrm{~L}$.

To obtain the third coating layer, $1.5 \mathrm{~L}$ of the twice-coated gibbsite particles in ethanol/water was brought into a three necked flask equipped with a stirrer. To the suspension, 65 $\mathrm{mL}$ of $10 \mathrm{w} / \mathrm{w} \%$ ammonia was added car efully and the solution was stirred for $15 \mathrm{~min}$. Subsequently, $5 \mathrm{~mL}$ of TEOS was added under the surface of the vigorously stirred solution. Stirring was stopped after a few minutes and the sample was ultrasonifcated. The ionic strength of the solution increases due to the hydrolysis of the TEOS. The high ionic strength makes the mixture unstable. Ultrasonification helps to prevent aggregation of particles. After 45 min the ultrasonification was stopped and stirring was started again for $4 \mathrm{~h}$. To obtain thicker silica coatings, new portions of TEOS can be added every $4 \mathrm{~h}$, following the same procedure.

2.3. Silica Coating: PVP Method. Poly(vinylpyrrolidone) (250 g, MW 40 000, PVP-40T, Sigma) was dissolved by alternating stirring and ultrasonification in $400 \mathrm{~mL}$ of demineralized water (total volume $\pm 500 \mathrm{~mL}$ ). To this viscous solution, $36 \mathrm{~mL}$ of a $56 \mathrm{mg} / \mathrm{g}$ gibbsite dispersion was added $(\approx 2 \mathrm{~g}$ of gibbsite). The mixture was slowly stirred for 1 day to let the PVP cover the surface of the gibbsite platelets. Next, it is diluted four times and centrifuged for 1 week at $740 \mathrm{~g}$ to remove the unabsorbed PVP. The coated gibbsite particles were easily redispersed in ethanol. To $243 \mathrm{~mL}$ of a $5 \mathrm{~g} / \mathrm{L}$ gibbsite dispersion was added $713 \mathrm{~mL}$ of ethanol sl owly. After 

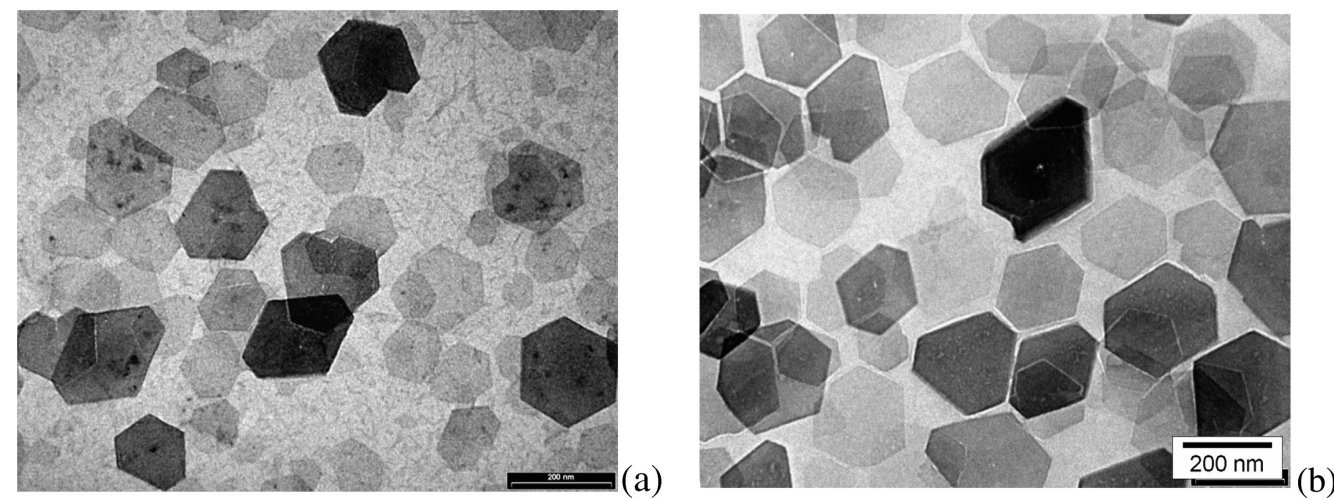

Figure 1. (a) TEM micrograph of gibbsite particles, average size $134 \mathrm{~nm} \pm 33 \%$. (b) TEM micrograph of gibbsite particles after centrifugation, average size $224 \mathrm{~nm} \pm 21 \%$.

10 min of stirring, ammonia was added in portions during 30 min. Finally, $20 \mathrm{~mL}$ of TEOS was added under the surface of the vigorously stirred solution. The reaction was completed in $24 \mathrm{~h}$. TEOS addition can be repeated after at least $4 \mathrm{~h}$ to obtain a thicker silica layer.

A generic problem of the coating with silica is the nucleation of silica particles. A simple but effective way to suppress the silica nucleation is increasing the ammonia concentration. Here, tests were done with ammonia concentrations of 46-73 $\mathrm{mL} / \mathrm{L}$ (only for the PVP method).

2.4. Characterization. A Tecnai 10 or 12 (FEI Co.) transmission electron microscope (TEM) operated at either 100 or $120 \mathrm{kV}$ was used to characterize the synthesized particles. Particle sizes were determined by measuring the projected area of at least 100 particles using an image-processing program. The angle-to-angle distance was calculated from the measured surface by assuming the particles are hexagons. Scanning electron microscopy (SE M) images were made on a Philips XL30F EG-scanning electron microscope operated at 10 $\mathrm{kV}$. Samples were sputter coated with $5 \mathrm{~nm}$ Pt/Pd to enhance the conductivity of the sample surface for SEM. The energy disperse X-ray analysis (EDX) was performed on a Tecnai 20 (FEI Co.) electron microscope operated in scanning TEM mode.

The solid content of the dispersions was determined from the difference between the mass of a portion of the dispersion before drying and the mass of the dried gibbsite.

\section{Results and Discussion}

3.1. Gibbsite Synthesis. On dissolving AIP and $\mathrm{ASB}$, the $\mathrm{pH}$ of the solution changed from 1 immediately after mixing to a $\mathrm{pH}$ value of $5-6$ after 7 days. AIP and ASB did not dissolve totally. The turbidity of the sample depends on the dissolution time (1-2 weeks), but a clear solution is not expected, since above an $\mathrm{Al} / \mathrm{Cl}$ ratio of 1.5, gibbsite is formed. ${ }^{33}$ The solid content of the dialyzed samples is $8-9 \mathrm{~g} / \mathrm{L}$. The used amount of $\mathrm{HCl}$ $(0.06 \mathrm{M})$ was accidentally too low, leading to a higher polydispersity; $0.09 \mathrm{M}(8.6 \mathrm{~g} / \mathrm{L})$ should have been used. TEM images of platelets after synthesis and 7 days dialysis look somewhat messy (Figure 1a). Between the gibbsite plates (diameter $134 \mathrm{~nm} \pm 33 \%$ ) smaller gibbsite particles and unidentified material is visible. The unidentified material might be boehmite or pseudoboehmite. ${ }^{34}$ As can be seen by comparing parts $a$ and $b$ of Figure 1, purification by centrifugation is successful. As the sediment redisperses easily in water, centrifuged particles are used for further experiments. Centrifuga-

(33) Buining, P. A.; Pathmamanoharan, C.; J ansen, J. B. H.; Lekkerkerker, H. N. W. J . Am. Chem. Soc. 1991, 74 (6), 1303-1307. (34) Antunes, M. L. P.; Santos, H. D.; Santos, P. D. Mater. Chem. Phys. 2002, 76, 243-249. tion not only removes small particles but also reduces the polydispersity of the sample by $30 \%{ }^{35}$ By X-ray diffraction of the dried centrifuged particles, the material is clearly proved to be gibbsite.

3.2. Silica Coating: Water Glass Method. For deposition of silica on gibbsite particles, a balance between the concentrations of silicate and gibbsite should be found. Too low a concentration of gibbsite stimulates the formation of silica particles; nucleating silicate should be able to precipitate on gibbsite and not form a particle itself. Too high a gibbsite concentration brings the risk of aggregation; the contacts between two gibbsite particles are made permanent by silica precipitation between them.

During the addition of the water glass solution, the $\mathrm{pH}$ of the gibbsite dispersion changes quickly from 6 to 10. In that period aggregation is also induced by a decrease of the surface potential, as the isoelectric point of the gibbsite (10) is approached. By adding water glass rapidly, in a sufficiently dilute dispersion, using ultrasonic treatment and stirring the dispersion, aggregation can be avoided and the best particles are obtained. ${ }^{21}$

Figure 2a shows a TEM picture of the once coated gibbsite particles. At high magnification the irregular surface of the particles is visible. This irregular surface must be the silica coating, since the uncoated gibbsite surface appears smooth under the TEM (Figure 1). Another indication of the presence of silica on the surface is the enhanced stability of the particles. Whereas gibbsite flocculates in ethanol, a dispersion of once-coated particles is stable for at least a few hours.

The covering layer consisting of silica particles nucle ating from water glass is now a few nanometers thick. ${ }^{21}$ For the next coating step, another method is used: TEOS is directly added to the alkaline, aqueous dispersion. The ethoxy groups of TEOS are hydrolyzed at the interface between TEOS and water. The silicic acid mol ecules leave the TE OS dropl ets and form oligomers that attach to the silica on the gibbsite. A TEM picture of twice-coated particles is shown in Figure $2 \mathrm{~b}$. The roughness of the particle surface varies between different batches of covered gibbsite. The roughness is influenced by the roughness of the first coating. When the hydrolysis of the TEOS is too fast, protrusions and small silica particles may be formed. Two black arrows indicate small protrusions in Figure $2 \mathrm{~b}$. In the same

(35) Wijnhoven, J . E. G. J . In preparation. 

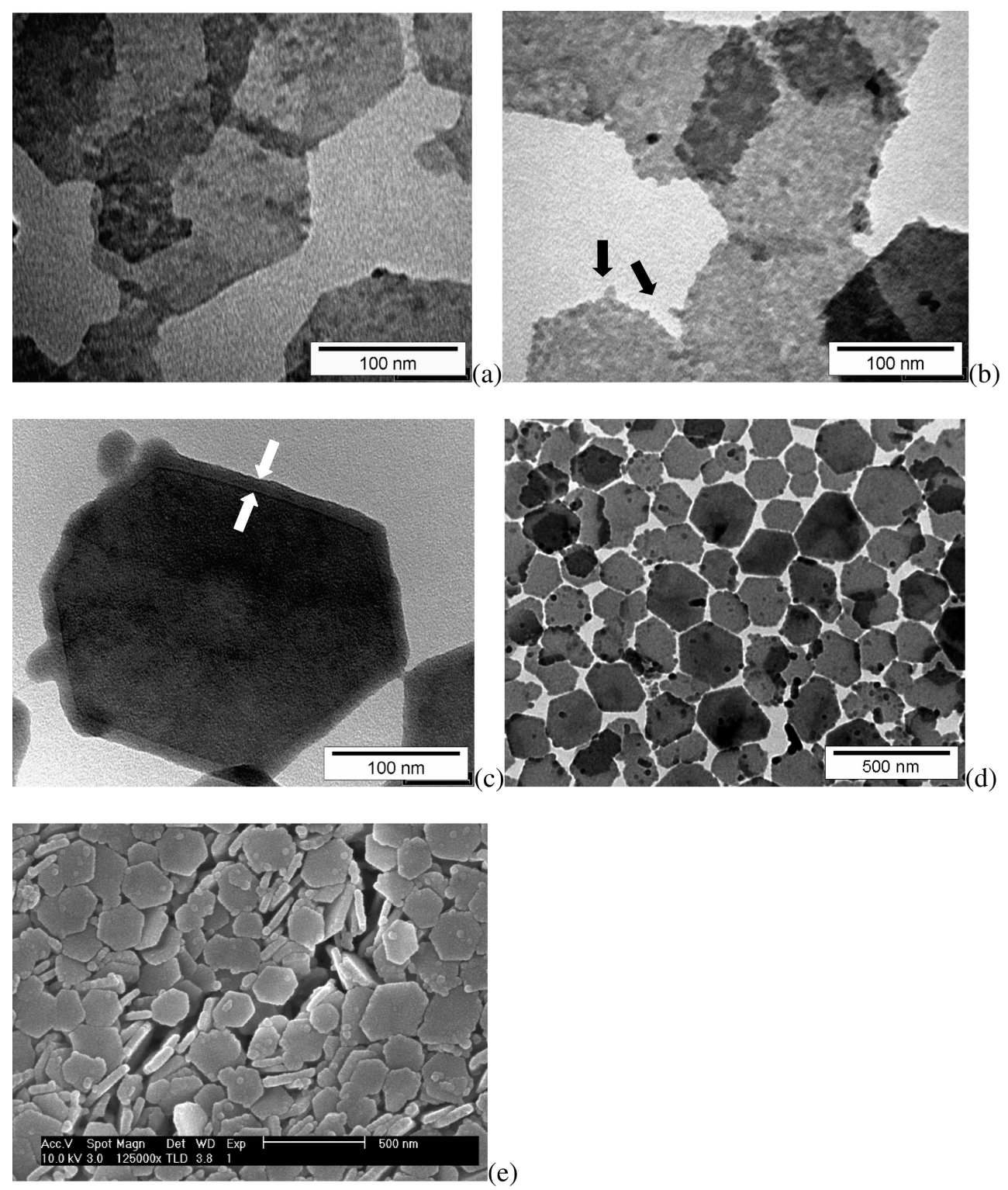

Figure 2. (a) TEM image of gibbsite particles covered with one layer of silica. Small silica particles have been precipitated from water glass and cause the roughness of the surface. (b) TEM image of twice silica-coated gibbsite particles. The surface is still rough. The black arrows point to extra silica growth. (c) TEM image of three layers of silica on gibbsite produce a smooth surface. Between the white arrows the silica layer (13 nm thick) is visible. The layer thickness is homogeneous. The silica covers the whole gibbsite particle. Extra silica growth is also visible. (d) TEM image of silica-coated gibbsite particles. Some loose silica spheres are present on and between the gibbsite. (e) SEM picture of silica-coated gibbsite particles. Small silica spheres are visible between the particles. Surface roughness is caused by the platinum/paladium coating which is necessary to get a SEM picture.

sample, up to 4 times larger protrusions are found. In other samples, no protrusions were found. Bearing two silica layers, the gibbsite is stable enough to be transferred into a mixture of water and ethanol (50/50, v/v) and ammonia. But if the silica layer is thick enough, it is also possible to work in ethanol. Figure 2c shows a gibbsite particle covered with three layers of silica. Between the white arrows the silica layer is visible; its thickness is $13 \mathrm{~nm}$. This means that the total thickness of the plates after coating with three layers of silica is $34 \mathrm{~nm}(2 \times 13+8 \mathrm{~nm}$ of the gibbsite core). Some silica particles are clustered on the surface of gibbsite. They are most likely formed in the second growth step in which the emulsifying of the TEOS was not good. Figure $2 d$ gives an overview with a number of particles. In comparison to pure gibbsite partides, as shown in Figure 1, the corners of coated particles are less sharp and the surface is rougher. On a SEM picture (Figure $2 e)$, the thickness of the plates is also visible, because some of the particles are standing on their sides. This sample was prepared by drying a concentrated solution of coated gibbsite in a sample cup. The picture is made from the bottom part of the sample. No longe-range ordering was visible, due to the size differences of the particles and the preparation method of the sample. Because of the particle's shape, there is some ordering; particles are either lying flat or standing on their sides.

The covering of gibbsite with silica by the water glass method described above is very good: all the particles are totally covered and the thickness of the silica layer is adjustable. Due to the higher isoelectric point of gibbsite compared to boehmite (where the method initially was developed for), the coverage with silica is more sensitive to the formation of aggregates. M oreover, 

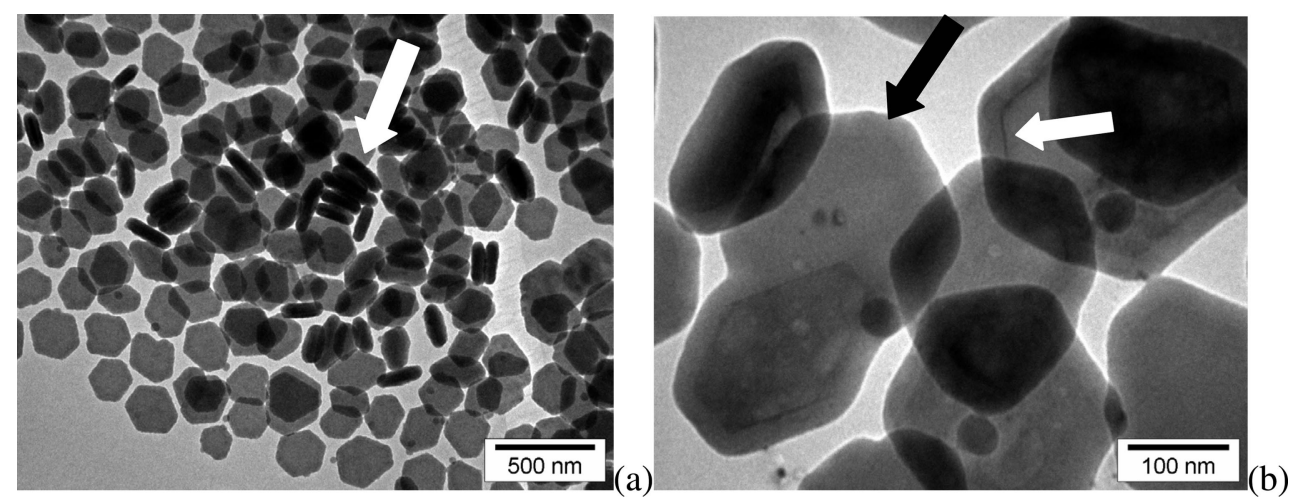

Figure 3. (a) TEM image of gibbsite particles coated with silica via the PVP method. The total thickness of a particle is $56 \mathrm{~nm}$. The white arrow points to a group of standing particles. (b) TEM image of gibbsite particles coated with silica via the PVP method. The white arrow points to the silica layer $(24 \mathrm{~nm})$. The black arrow points to two aggregated gibbsite platel ets covered and joined with silica.
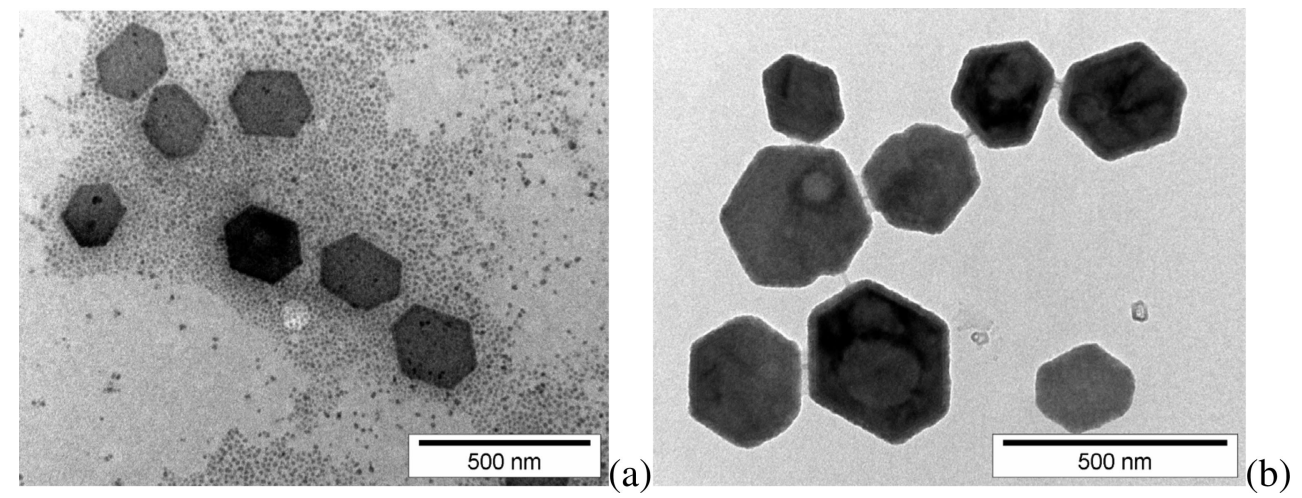

Figure 4. (a) EM image of silica-coated gibbsite particles (PVP method). The ammonia concentration (46 mL/L) is too low: silica particles are nucleated in the solution. (b) TEM image of silica-coated gibbsite particles (PVP method). Proper ammonia concentration $(62 \mathrm{~mL} / \mathrm{L})$ : no silica particles or aggregated gibbsite particles are produced.

due to dialysis and centrifugation steps taking a few days, the method is quite time-consuming.

3.3. Silica Coating: PVP Method. Graf et al..$^{32}$ describe the mixing of PVP with different types of particles as a stabilizing agent before Stöber growth of silica. Their method produces smooth, nicely coated gibbsite particles but is only described for very dilute $(0.08 \mathrm{~g} / \mathrm{L})$ systems. We el aborated the method to be able to obtain a workable amount of coated particles per volume. Figure 3a shows a TEM picture of gibbsite particles coated with silica via PVP. At some parts of the picture, the concentration of gibbsite is quite high. Particles are lying on top of each other. They are still single particles, not aggregates. (In this method, aggregates are mainly formed during the centrifugation step, before the coating. So aggregates are covered as a whole with silica. Here every particle is totally coated.)

The silica coating on the particles is quite thick, 24 $\mathrm{nm}$. The particles in the middle of the picture, indicated by a white arrow, can easily stand on their sides because the sides are $56 \mathrm{~nm}$. The diameter of these particles is $230 \mathrm{~nm}$ (including the coating). The aspect ratio (diameter/thickness) of the particles is 4 . Before coating the aspect ratio was 23 . Figure $3 \mathrm{~b}$ shows another part of the same sample at higher magnification. The silica coating is clearly visible and indicated with a white arrow. The whole particle is evenly coated with silica. The coating seems very nice and smooth. The black arrow points to an aggregate. Aggregation sometimes takes place during the centrifugation of the particles after PVP coating. That is what has happened here, since the silica coating is formed around the two particles together. Aggregated particles are occasionally observed. Aggregation can almost be prevented by careful centrifugation.

The centrifugation and removal of PVP is not strictly necessary in very diluted systems. ${ }^{32}$ But direct addition of TEOS to the concentrated, viscous system we use leads to aggregation due to bad mixing. When added, the TEOS is not able to precipitate quickly on gibbsite particles, because the mixture is too viscous. Consequently, large aggregates of particles are formed which sediment when stirring is stopped.

In Stöber synthesis an ammonia concentration of 40 $\mathrm{mL} / \mathrm{L}$ is normally used. As can be seen from Figure $4 a$, a low ammonia concentration causes second nucl eation of silica particles. The coating layer is also thinner than expected, because a big part of the TEOS is grown into the silica particles. It is known ${ }^{36}$ that a higher ammonia concentration suppresses the second nucleation in growing silica layers around silica spheres. Ther efore, several ammonia concentrations are tested. A few characteristic results are shown in Figure 4. A value of $62 \mathrm{~mL} / \mathrm{L}$ (F igure 4b) gives the best result. Higher concentrations of ammonia favor the formation of aggregates, al though a lot of nicely coated particles are also seen in such samples.

(36) van Blaaderen, A.; Kentgens, A. M. P. J . Non-Cryst. Solids 1992, 149, 161-178. 


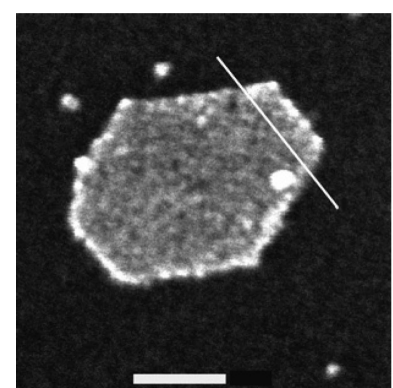

(a)

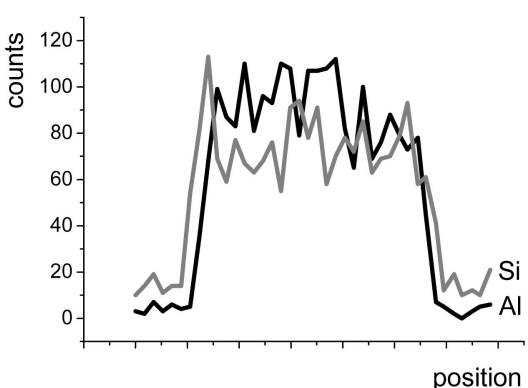

(b)

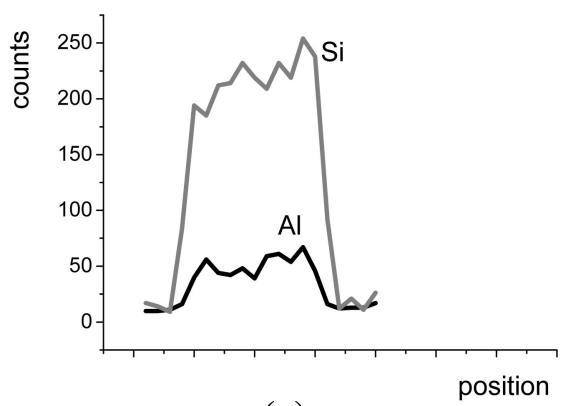

(c)

Figure 5. (a) Scanning TEM of silica-coated gibbsite particle. The white line corresponds to the measurements shown in Figure 4b. The scale bar is $50 \mathrm{~nm}$. (b) Silicon and al uminum signal of EDX measurement on a twice-coated gibbsite particle (water glass method). The silicon and aluminum signals from the faces are of the same intensity. (c) Silicon and aluminum signals from an EDX measurement on a gibbsite particle coated with a $10 \mathrm{~nm}$ thick silica layer (PVP method).

Table 1. Ratio of the Counts of Silicon and Aluminum for Gibbsite Coated with Silica Layers of Different Thicknesses

\begin{tabular}{cc}
\hline particle type & counts Si/counts Al \\
\hline one silica layer (water glass) & 0.3 \\
two silica layers (TES growth) & 1 \\
three silica layers (Stöber growth) & 4 \\
silica coating by PVP & 7
\end{tabular}

The silica layer is visible on the TEM pictures. With $X$-ray diffraction the presence of the silica layer cannot be proved because the silica is amorphous. Therefore, EDX is used to prove the presence of silicon (and thus silica). During scanning TEM, X-rays emitted by aluminum and silicon are detected. In Figure $5 a$, the white line shows the part of a particle that has been scanned. Figure $5 b, c$ shows two typical signals; part b shows a twice-coated particle and part c a PVP-coated particle with a thick silica layer. The X-ray signal from silicon starts to increase before the aluminum signal and decreases after because the silicon is on the outside of the gibbsite. The silicon-aluminum ratio increases by increasing silica thickness, as is shown in Table 1.

\section{Potential Use}

Silica-coated particles are good candidates for a wide area of applications; three of them are discussed in this section.

4.1. Hollow Particles. Acid leaching of aluminum is a common technique in catalysis. ${ }^{37-39}$ By acid leaching, mesopores are formed and the Al/Si ratio can be adjusted. For zeolites the $\mathrm{Al} / \mathrm{Si}$ ratio is an important parameter that exerts a strong influence on properties such as ion exchange capacity or catalytic activity and selectivity. Here, the acid-leaching technique is used to prepare hollow silica platelets based on gibbsite. Whereas preparation of hollow silica spheres ${ }^{40-42}$ and silica nanotubes ${ }^{43}$ has been pursued extensively the

(37) Beyer, H. K. Mol. Sieves 2002, 3, 203-255.

(38) Tromp, M.; van Bokhoven, J . A.; Oostenbrink, M. T. G.; Bitter, J. H.; de J ong, K. P.; Koningsberger, D. C. J . Catal. 2000, 190, 209214.

(39) van Donk, S.; J anssen, A. H.; Bitter, J . H.; de J ong, K. P. Catal. Rev. Sci. Eng. 2003, 45, 297-319.

(40) Shchukin, D. G.; Sukhorukov, G. B.; Mohwald, H. Angew. Chem. Int. Ed. 2003, 42, 4472-4475.

(41) Imhof, A. Langmuir 2001, 17, 3579-3585.

(42) Caruso, R. A.; Susha, A.; Caruso, F. Chem. Mater. 2001, 13, 400-409.

(43) Zygmunt, J .; Krumeich, F.; Nesper, R. Adv. Mater. 2003, 15, 1538-1541. last years, hollow platelets are not yet known. The silicacoated gibbsite platel ets are an excellent starting point to obtain hollow platelets: Gibbsite is known to be soluble in strong acids, whereas silica is not. Figure 6 shows pictures of particles after acid leaching for 3 days. In Figure 6a the white arrow points to a hollow particle. On electron microscopy images, hollow particles are recognizable by a light core with dark edges, whereas filled particles are homogeneous. Because the middle of the particles contains less material than the walls, more electrons go through the sample, leading to a lighter color on the image. The black arrow points to a particle that still contains gibbsite. The particle has a homogeneous color on the picture. Whether the gibbsite is not etched away because of a too short leaching period or other reasons (e.g. a too low permeability of the silica layer) is still a point of investigation. In Figure $6 b$ an aggregate of particles is shown. In the aggregate some particles are standing on their sides. The white arrows point to these particles and it is clearly seen that they are empty inside. In Figure $6 c$, the black line indicates the position of EDX measurements on a hollow particle. Figure $6 \mathrm{~d}$ presents the results. At the moment the electronbeam enters the sample, a signal from silicium is detected. A very small amount of al uminum is detected; the remnant of the gibbsite. (The ratio between the number of counts for silicon and alumina is 15 , much higher than for a particle still containing gibbsite (Table 1). Moreover, the thickness of silica layer of the hollow particle is $13 \mathrm{~nm}$ compared to $25 \mathrm{~nm}$ for the gibbsite-containing particles.) The number of counts for the silicium signal is larger at the edge of the particle due to the thicker silica layer at the edges.

4.2. Fluorescence. Coupling of fluorescent dyes to a silane-coupling agent allows their incorporation in silica. The possibility to incorporate a dye in a silica layer is used to visualize silica particles in a confocal microscope ${ }^{44,45}$ or to study their dynamic behavior. ${ }^{46-48}$ The incorporation of dye has been applied successfully for silica spheres ${ }^{18}$ and for silica-coated boemite rods. ${ }^{21}$

(44) van Blaaderen, A.; Imhof, A.; Hage, W.; Vrij, A. Langmuir 1992 8, 1514-1517.

(45) Verhaegh, N. A. M.; van Blaaderen, A. Langmuir 1994, 10.

(46) Imhof, A.; Dhont, J . K. G. Colloids Surf., A 1997, 122, 53-61.

(47) van Bruggen, M. P. B.; Lekkerkerker, H. N. W.; Maret, G.; Dhont, J. K. G. Phys. Rev. E 1998, 58, 7668-7677.

(48) Kluijtmans, S.; Dhont, J . K. G.; Philipse, A. P. Langmuir 1997, 13, 4982-4987. 

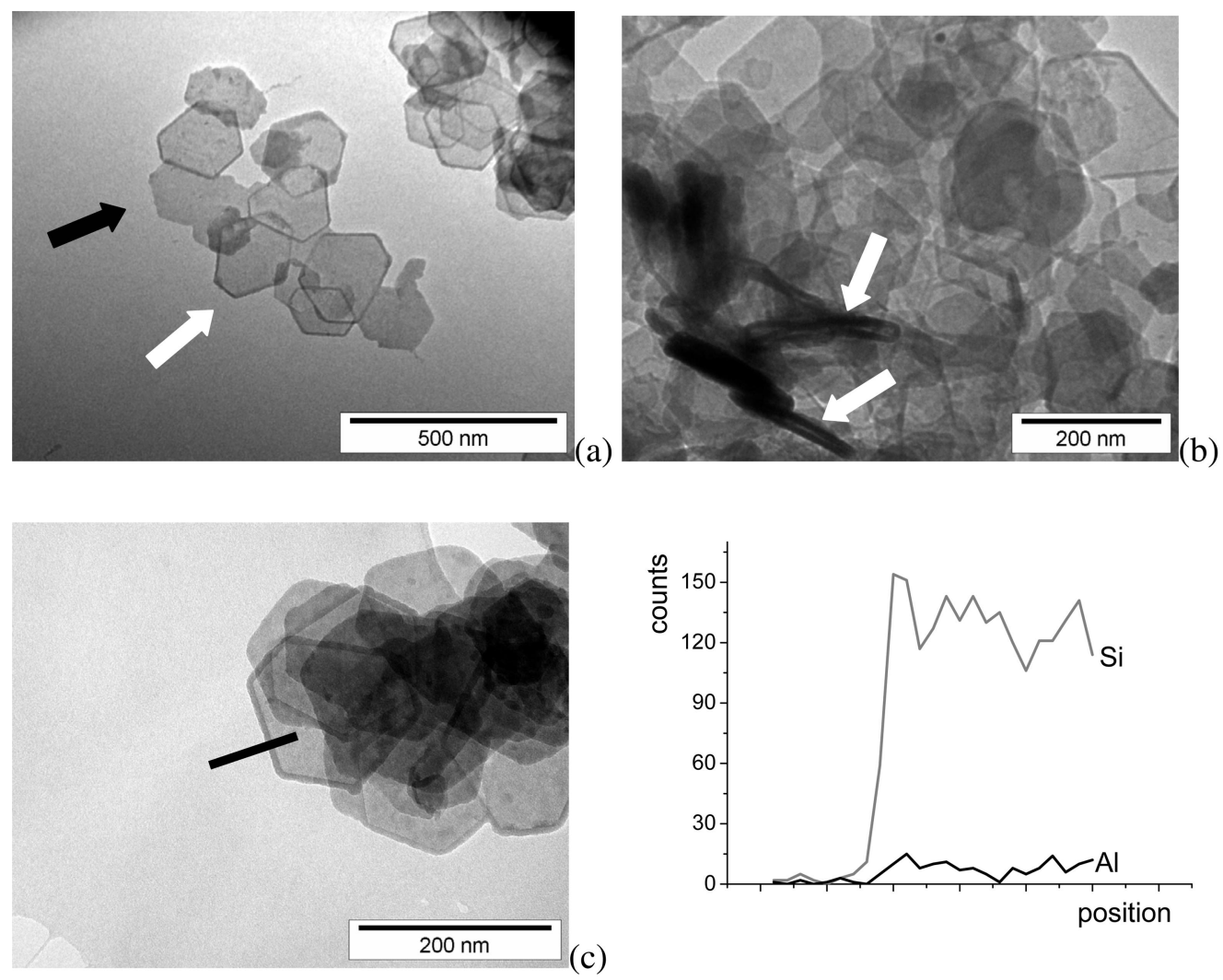

Figure 6. (a) TEM image of acid-leached particles. The white arrow points to a hollow silica particle, and the black arrow points to a silica-coated gibbsite particle. (b) TEM image of a group of aggregated particles; two particles, pointed to by a white arrow, are standing on their sides and are clearly empty. (c) The black line indicates where the EDX measurements of part $d$ are performed. (d) Result of an EDX measurement, where there is hardly any signal for aluminum: the acid leaching was successful.

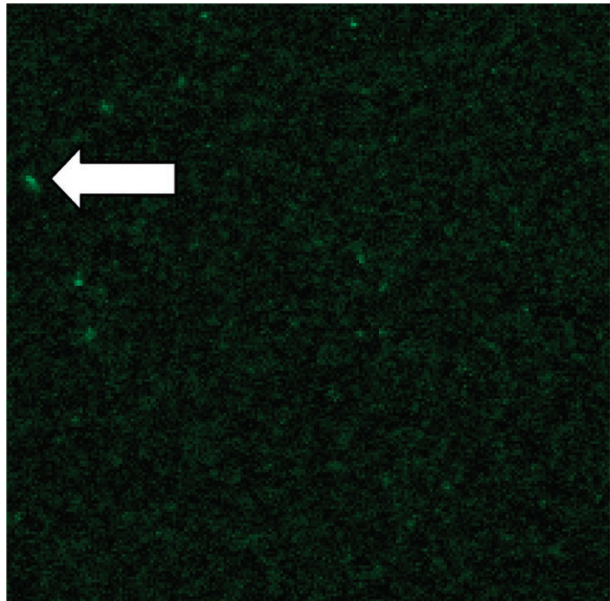

Figure 7. Confocal microscopy image of fluorescently labeled silica-coated platelets, size of the view $60 \times 60 \mu \mathrm{m}$, the arrow points to an aggregate.

A first attempt indicates that it is also possible to incorporate dye in the silica layer around a gibbsite particle. This opens up opportunities for studies of the structure and dynamics of platelike systems with the confocal microscope or with the fluorescence recovery after photobleaching (FRAP) technique. In Figure 7 a confocal microscope image of gibbsite platel ets is shown. The arrow points to an aggregate of particles (bright color). In this picture it is not possible to see individual particles because they are too small. Several pictures taken at the same place can be put together into a movie. In such a movie it is clear that particles are moving separately.

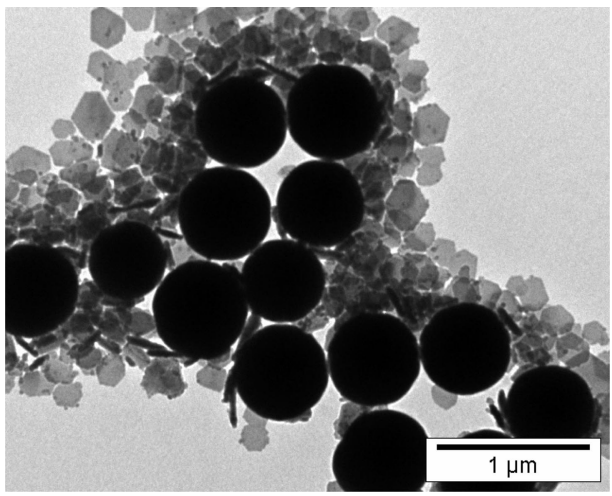

Figure 8. TEM image of a mixture of silica spheres (radius $350 \mathrm{~nm}$ ) and silica-coated gibbsite platelets.

4.3. Mixtures. Mixtures of rods and spheres are studied thoroughly. Both the theory ${ }^{49}$ and the experiments ${ }^{50}$ on the phase behavior have been described. Small amounts of (silica coated) rods do assemble the silica spheres rapidly, leading to aggregates that crystallize. Recently, a theory on the interaction and the phase behavior of mixtures of plates and spheres has been proposed. 51,52 The experimental study of mixtures of silica spheres and gibbsite plates is, just as for rods and spheres, not directly possible. Their isoelectric

(49) Vliegenthart, G. A.; Lekkerkerker, H. N. W. J . Chem. Phys. 1999, 111, 4153-4157.

(50) Vliegenthart, G. A.; van Blaaderen, A.; Lekkerkerker, H. N. W. Faraday Discuss. 1999, 112, 173-182.

(51) Oversteegen, S. M.; Lekkerkerker, H. N. W. Phys. Rev. E 2003 68.

(52) Oversteegen, S. M.; Lekkerkerker, H. N. W. J . Chem. Phys. 2004, 120, 2470-2474. 
points differ too much ( $\mathrm{pH} 2$ and 9). At neutral $\mathrm{pH}$ the particles would attract each other and thus from aggregates. With silica coating of the plates this problem can be overcome. The phase behavior of mixtures of these model particles will be studied. A first step to the practical systems such as paints were pigment particles that do have different shapes.

\section{Summary and Conclusion}

Gibbsite can be covered with silica by both described methods. Coating by the water glass method is timeconsuming. The silica covers the whole gibbsite particle and the surface is in the first two steps rough. By adding thicker silica layers, the surface becomes smoother. Aggregation is sometimes a problem. The PVP method works very well and is, at the same time, less time consuming. The coating of the gibbsite with PVP is easily done. Only layers of a few nanometer thick do not cover the whole particle. For those thin layers the water glass method (only the first step) is appropriate. The silica-coated particles are stable in ethanol and do have the same surface properties as pure silica particles. It may be assumed that particles can undergo the existing silica modi fications such as coating with apolar chains. By adding the proper amount of ammonia, the second nucleation of silica can be avoided.

Acknowledgment. J . D. Meeldijk (Debije Institute, Utrecht University) is thanked for help with the electronmicroscopes and for performing the EDX measurements. M. Versluys-Helder (Inorganic Chemistry, Utrecht University) is thanked for performing X-ray diffraction. C. Vonk is thanked for sharing her results on the ammonia concentration and for the pictures of Figures 4 and 7. R. P. A. Dullens is thanked for taking the SEM image for Figure 2. S. M. Oversteegen and P. $M$. J ohnson are thanked for the critical reading of the manuscript. Prof. H. N. W. Lekkerkerker is acknowledged for support and encouragement. This work was financially supported by the "Nederlandse organisatie voor Wetenschappelijk Onderzoek" (NWO).

CM 049437M 